\title{
Structure Design and Kinematics Analysis for a New-type All-electric Sweeper
}

\author{
Xiaoqin Yin ${ }^{1, a^{*}}$ Linji Zhu ${ }^{2, b}$ \\ ${ }^{1}$ Institute of Intelligent Machines and Robotics, Jiangsu University, Zhenjiang 212013 China \\ ${ }^{2}$ Institute of Intelligent Machines and Robotics, Jiangsu University, Zhenjiang 212013 China \\ akyd_black@163.com, ${ }^{\text {b }}$ kyd_black@163.com
}

Keywords: small-sized all-electric sweepers; the third sweeping brush; Denavit-Hartenbergn method; Monte-Carlo principle.

\begin{abstract}
This paper is meant to put forward and design the third sweeping brush structure which can clean curbstones, corners of walls and etc., optimizing the cleaning structure of the domestic small-sized all-electric cleaning vehicle. The new structure can expand the cleaning area the original sweepers cover, thus improving the cleaning efficiency and reducing human resources investment. To begin with, considering the functions and requirements of the third sweeping brush, the paper firstly designs several structures of some sweeping brushes and determine the ultimate structure type after analyzing their advantages and disadvantages. Next, using Denavit-Hartenbergn method, the paper establishes the kinematics models of the sweeping brush and solves its kinematics equation through homogeneous coordinate conversion. Finally, based on Monte-Carlo principle, the paper depicts the working space of the sweeping brush by using MATLAB on the basis of kinematic equation. The brushing dots are closely and equally distributed, therefore, the sweeping brush can meet the working requirements.
\end{abstract}

\section{Introduction}

With the constant development of society, the environmental problems are increasingly paid attention to. However, there are many problems lying in the manual cleaning, for example, flying dust, which exerts serious influence on the cleaners' health and causes secondary pollution on the environment unavoidably ${ }^{[1-3]}$. Therefore, mechanized cleaning has gradually penetrated into people's life.

Compared to the good-sized sweepers, the small-sized one, as a new-type environmental protection equipment, is especially applicable for cleaning the densely-populated roads or the roads whose condition is complicated, such as streets, parks, campuses, squares, residential lots and etc.. Due to its flexible and convenient operation, the small-sized sweepers have great prospects for development ${ }^{[4,5]}$. However, there are distinct limitations existent in domestic small-sized sweeper is limited in their cleaning scope ${ }^{[6,7,8]}$. Hence, some human input is required to clean the curbstone, the areas below the public chairs, corners of walls and other hygienic dead angles. To solve this problem, the paper has designed a new-type sweeper structure. Specifically, on the basis of the two sweeping brushes, the third sweeping brush is added, whose length, angle of rotation and pose can be adjusted to achieve the function of hygienic dead angles cleaning. 


\section{Structure Design of the Sweeping Third Brush}

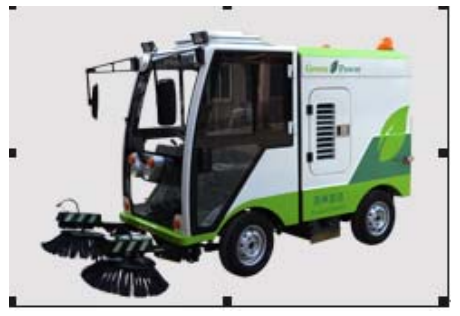

(a)

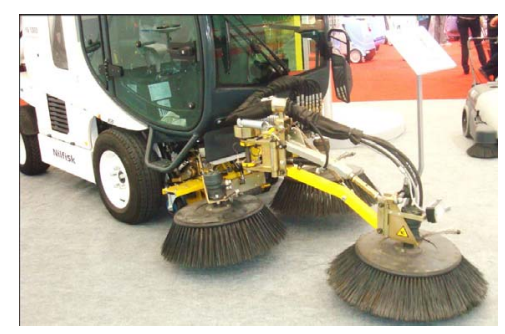

(b)

Fig.1 typical structure of small-sized sweeper

As is shown in Fig.1(a), most of the small-sized all-electric sweepers on current domestic market are suction type. Their side-brushes, relatively fixed to the sweeper's body, can rotate on its own axis. After the rotation, the rubbish can be gathered to suction nozzles which then suck them into the rubbish bin and complete the cleaning process ${ }^{[6,7]}$. However, the cleaning scope of the sweepers of this type is quite limited, for example, it cannot clean the curbstone, the areas below the public chairs, corners of walls and etc.. This paper, learning from the structure of the small-sized sweepers in Europe and America (as is shown in Fig.1(b), designs the third sweeping brush which is placed in the middle of the side-brushes. The main structure of the third sweeping brush is similar to human's arm. Driven by its shoulder joint, it can rotate up and down(left and right); driven by its elbow joint, it can control the length of Its arm; driven by its wrist, it can adjust the pose of its disc brush. Because of the coordinating control, the third brush can clean the curbstone by elevating its arm, clean the areas below the public chairs by stretching its arm, clean the corners of walls by flipping certain angles and etc..

To conclude, the structure of the third sweeping brush should be simple rather than too complicated under the premise of functioning perfectly. The functioning area should cover the width of the pavement on the curbstone and the control system can be combined with the original electric sweepers.

\section{Mechanism Selection of the Third Sweeping Brush}

The third sweeping brush of the small-sized all-electric sweepers is similar to the human's arm. The first three joints are used to send the wrist of the robot arm to the assigned position. Learning from the configuration of the industrial robot and considering the function and requirement of the third brush, this paper chooses the articulated manipulator as the main configuration of the third brush's arm.

The whole arm needs to swing in the plane of xoy,xoz,yoz and rotate around the axis z. According to the calculation formula of open chain spatial degree of freedom ' $F=\sum_{i=1}^{P} f_{i}$, (F is the DOF of mechanism and $f_{i}$ is the DOF of each joint) ${ }^{[9,10]}$, the degree of freedom of brush is 4 , so the mechanisms of brush have designed in three plan as is shown in Fig.2

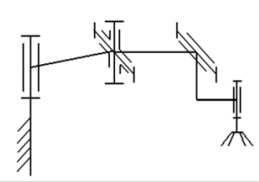

(a)

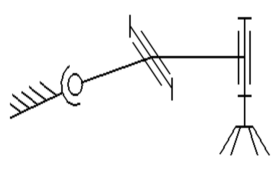

(b)

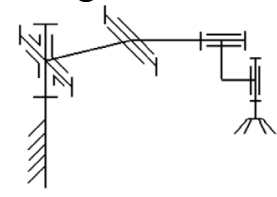

(c)

Fig. 2 structure of the third brush

As is shown in Fig. 2(a), the first joint is rotary joint, which can make the sweeping brush swing in the horizontal plane. The second joint is hook hinge, which can further expand the working space of its forearm in the horizontal plane and make its forearm swing in a certain range in the vertical plane. The third joint, also the rotary joint, which is used to flip the angle of the brush disc during the 
working, can make the sweeper function on the incline. When working, the brush disc, as a rotational input, can enlarge the cleaning areas of roads and clean the curbstones at both sides of roads, even the places below the public benches and the corners of walls. When stopping working, the third brush can be folded up to save space. Mainly composed of rotary joints and with simple structure and large functioning space, the robot arm is easy to control.

As is shown in Fig. 2(b), the mechanism configuration is spherical coordinate robot arm. The first joint, composed of spherical joints whose three freedoms make the brush can swing in three planes, undertakes the main working responsibility of the brush. The second one is oratory joint, which can make the brush disc further function higher or lower. Although the feature of the mechanism is less joints, high accuracy of working and a wide up-and -down range of swing, the working ability of spherical joints limits the left-and-right swing angle of the mechanism and its three freedom is hard to control.

As is shown in Fig. 2(c), the hook hinge, placed at the first joint, control the working condition of the brush in the both horizontal and vertical plane. The second joint can make the brush disc further function higher or lower. The third one can clean the sloping curbstones and roads by rotating around its forearm to adjust the pose of the brush discs. The left-and-right sweeping range of the brush is much smaller than project 2(a).

All the structures and features of the three projects and the result of the comparison concerning cleaning and recycling process considered, Fig. 2(a) is the ultimate sweeping structure.

\section{Kinematic Analysis of the Third Sweeping Brush}

The forward kinematic parse is gotten by using 'Denavit-Hartenberg method' ${ }^{[10,11,12]}$ and the frame of axes for link rod is established as is shown in Fig.3. Hooke hinge can be seen as two rotary joints and the parameter of each joint is showing in Tab.1. In the Tab.1, $\alpha_{i-1}$ is the angle that $z_{i-1}$ rotate around $z_{i}$ in the direction of $x_{i-1}$ and $a_{i-1}$ is the distance between $z_{i-1}$ and $z_{i}$ in the direction of $x_{i-1}$ and $\theta_{i}$ is the angle that $x_{i-1}$ rotate around $x_{i}$ in the direction of $z_{i-1}$ and $d_{i}$ is the distance between $x_{i-1}$ and $x_{i}$ in the direction of $z_{i-1}$. The four parameters determine the brush's position. The final transformation matrix is gotten after transforming 4 times which showing in followings.

$$
\boldsymbol{M}_{(i-1) i}=\operatorname{Rot}\left(x_{i-1}, \alpha_{i-1}\right) \operatorname{Trans}\left(x_{i-1}, a_{i-1}\right) \operatorname{Trans}\left(z_{i-1}, d_{i}\right) \operatorname{Rot}\left(z_{i-1}, \theta_{i}\right)
$$

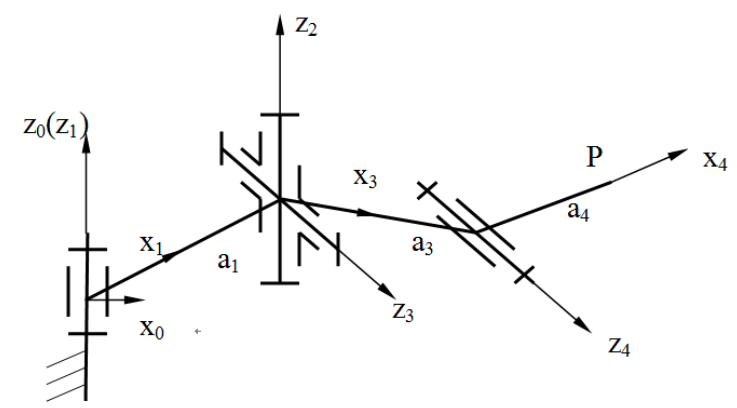

Fig.3 Frame of axes of the third brush

Tab.1 D-H parameter of the third brush

\begin{tabular}{rrcccc}
\hline joints $i$ & $\alpha_{i-1}\left({ }^{\circ}\right)$ & $a_{i-1}(\mathrm{~mm})$ & $d_{i}(\mathrm{~mm})$ & $\theta_{i}\left({ }^{\circ}\right)$ & angle range of joints $\left(^{\circ}\right)$ \\
\hline 1 & 0 & 0 & 0 & $\theta_{1}$ & $-45 \sim 45$ \\
2 & 0 & $a_{1}$ & 0 & $\theta_{2}$ & $-45 \sim 45$ \\
3 & 90 & 0 & 0 & $\theta_{3}$ & $-20 \sim 30$ \\
4 & 0 & $a_{3}$ & 0 & $\theta_{4}$ & $-45 \sim 30$ \\
\hline
\end{tabular}


$\theta_{1}$ and $\theta_{2}$ are the angles brush brushing in the horizontal plane around z-axis and the certain values showing in tab. 1 are determined according the working requirements. Moreover $\theta_{3}$ and $\theta_{4}$ are the angles rotating around z-axis in vertical plane, whose values are also in Tab.1.

Putting each value in Formula 1, the transformation matrix of each joint is gotten under followings.

$$
\begin{aligned}
& \boldsymbol{M}_{01}=\left[\begin{array}{cccc}
c_{1} & -s_{1} & 0 & 0 \\
s_{1} & c_{1} & 0 & 0 \\
0 & 0 & 1 & 0 \\
0 & 0 & 0 & 1
\end{array}\right] \quad \boldsymbol{M}_{12}=\left[\begin{array}{cccc}
c_{2} & -s_{2} & 0 & a_{1} \\
s_{2} & c_{2} & 0 & 0 \\
0 & 0 & 1 & 0 \\
0 & 0 & 0 & 1
\end{array}\right] \\
& \boldsymbol{M}_{23}=\left[\begin{array}{cccc}
c_{3} & -s_{3} & 0 & 0 \\
0 & 0 & -1 & 0 \\
s_{3} & c_{3} & 0 & 0 \\
0 & 0 & 0 & 1
\end{array}\right] \quad \boldsymbol{M}_{34}=\left[\begin{array}{cccc}
c_{4} & -s_{4} & 0 & a_{3} \\
s_{4} & c_{4} & 0 & 0 \\
0 & 0 & 1 & 0 \\
0 & 0 & 0 & 1
\end{array}\right]
\end{aligned}
$$

$c_{i}=\cos \theta_{i}, \quad s_{i}=\sin \theta_{i}$

The transformation matrix from 0 frame-of-axes to 4 is as following,

$$
\boldsymbol{M}_{04}=\boldsymbol{M}_{01} \boldsymbol{M}_{12} \boldsymbol{M}_{23} \boldsymbol{M}_{34}=\left[\begin{array}{cccc}
c_{12} s_{34} & -c_{12} s_{34} & s_{12} & a_{1} c_{1}+a_{3} c_{12} c_{3} \\
s_{12} c_{34} & -s_{12} s_{34} & -c_{12} & a_{1} s_{1}+a_{3} s_{12} c_{3} \\
s_{34} & c_{34} & 0 & a_{3} s_{3} \\
0 & 0 & 0 & 1
\end{array}\right]
$$

According to Fig.3, the coordinate of point $\mathrm{p}$ in 4 frame-of-axes is $\boldsymbol{P}_{4}=\left(a_{4}, 0,0\right)^{\mathrm{T}}$, which in 0 frame-of-axes is as following.

$$
\boldsymbol{P}_{0}=\boldsymbol{M}_{04} \cdot \boldsymbol{P}_{4}=\left(\begin{array}{c}
a_{1} c_{1}+a_{3} c_{12} c_{3}+a_{4} c_{12} c_{34} \\
a_{1} s_{1}+a_{3} c_{3} s_{12}+a_{4} s_{12} c_{34} \\
a_{3} s_{3}+a_{4} s_{34}
\end{array}\right)
$$

If knowing the parameters $a_{i}$ of the third brush and the driving angle $\theta_{i}$, the position coordinate of point $p$ in wrist joint can be solved. This progress is the analysis of forward kinematical parse, which is the basement of kinematic analysis of the third brush and the key to knowing the work space of the brush.

\section{The analysis of the third brush's working space}

The scale of the third brush's working space determines its working range, thus it is an important indicator to evaluate its working performance. There are many ways to analyze the working space of robot arms, of which graphic method ${ }^{[13]}$, numerical method and simulation ${ }^{[14]}$ method are commonly used. The graphic method, used to depict the working space boundary of the robot arm, has strong visualization, but the drawing is quite complicated. The numerical method is used to calculate the coordinates of the feature points on the working curved surface of robot arm and then the point-cloud composed of the feature points of undergoes the process of boundary curve fitting. Then the curves composed of point-clouds can depict the working space boundary after surface fitting. The typical method of numerical method includes Monte Carlo method, iteration method and search method. The numerical method can be used to compute and address graphics and images to obtain the workspace with the assistance of computer. Therefore, it can depict the workspace intuitively. The simulation method is used to construct a model for the objects of study in the MATLAB and other drawing softwares. After that, drivers are added to the each joint and the coordinates of the output end are recorded through the position sensor. This method does not involve solving the equation of the robot movement, so it is applicable for the structure which is complicated and has a large number of DOF. 
This paper analyzes the workspace of the sweeping brush with the assistance of Monte-Carlo method. Monte-Carlo ${ }^{[15]}$ is a method used to solve mathematic problems with the help of random numbers in accordance with some mathematic laws on the basis of probability statistics. When it is applied to obtain the workspace, the feature points $\mathrm{p}$ gather in the space where they may appear. Among the value range of each joint, the coordinates are came randomly. To solve the working space, all of the random points form a cloud chart and the boundary points are formed as the boundary lines which are also formed as the boundary surface.

According to the parameter of the selected sweepers , the values are set as $a_{1}=950 \mathrm{~mm}, a_{3}=600 \mathrm{~mm}$, $a_{4}=400 \mathrm{~mm} .10000$ random points are produced in MATLAB during the range of driving angles, which constitute the working space chart under the operation of forward kinematical parse in MATLAB. The graphic model and each plane of projection are showing in Fig.4, Fig.5, Fig.6, Fig.7.

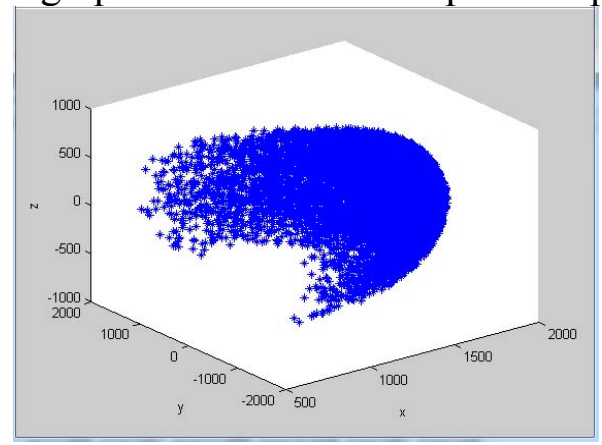

Fig.4 Graphic model of working space

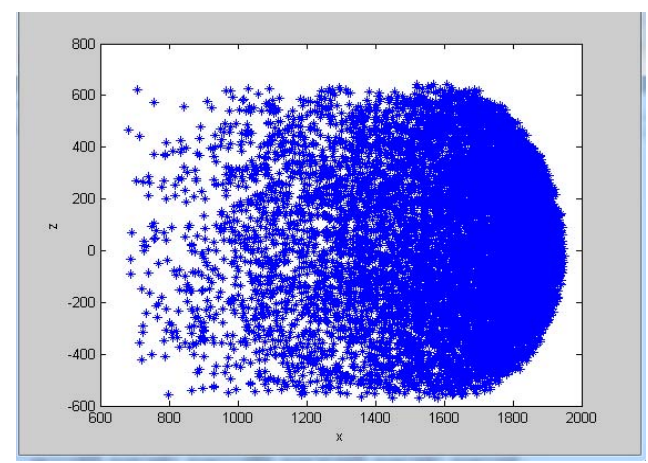

Fig.6 projection plane in surface $\mathrm{xoz}$

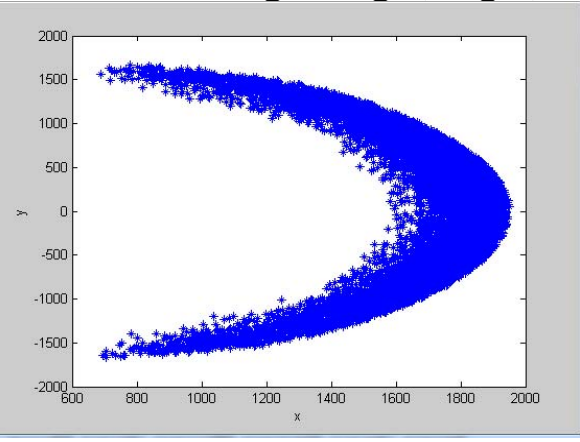

Fig.5 projection plane in surface xoy

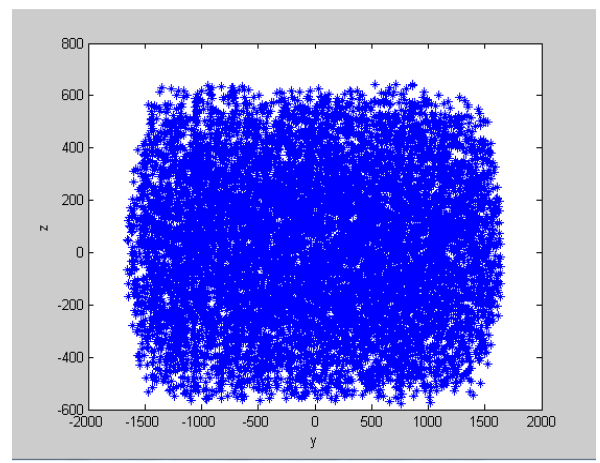

Fig. 7 projection plane in surface yoz

From Figure4 to Figure7, it can be seen that the workspace of the sweeping brush is similar to a fan cylinder. The projection of the horizontal plane is part of a sector. The cleaning surface of the original sweeper is a rectangle whose width(about $1.8 \mathrm{~m}$ ) is the distance between two side-brushes. From Figure 5, it can be learned that the cleaning scope is obviously enlarged, compared to the original one, and more specifically, the width of the sweeping horizontal surface(about $3.5 \mathrm{~m}$ ) is three times as long as the width of the original one. The projection of H-perpendicular plane (xoz plane) and the V-perpendicular plane(yoz plane) presents the deviation of the numerical direction of the sweeping brush. Therefore, the third sweeping brush can function higher of lower flexibly to clean the curbstones whose height reach $600 \mathrm{~m}$, the places below the public benches or even the front and lateral sloping roads.

\section{Summary}

This paper designs and improves the third sweeping brush based on the structure of the original small-sized all-electric sweepers. Firstly, the main structure of the third brush is selected and designed and the problem of the positional forward solution is solved with D-H coordinate transformation method. Secondly, on the basis of that, the workspace of the brush is depicted with the numerical method, from where its cleaning ability and superiority can be clearly seen. Therefore, it provides the guidance for the research and development domestic small-sized all-electric sweepers. 


\section{References}

[1] Mark. Leister, The development trend of global road sweeper, J. Special Vehicle. 2008 91-93.

[2] Henan Ge, Current situation of national road sweeper industry and its development trends, J.Construction machinery, 11 (2004) 54-56. (In Chinese)

[3] Wenxing Ma, The development of sweeper, Chemical industry press, Beijing, 2006, pp. 94-98. (In Chinese)

[4] Jiangtao Lee, The development situation and trends of national sweeper, J. Construction machinery technology and management. 4 (2000) 16-17. (In Chinese)

[5] Qiming Zhang, The development trends of road sweeper, J. Construction machinery and equipment. 825 (2008) 43-44. (In Chinese)

[6] Leiqing Zhou, The situation and application of national sweeper, J. Municipal facilities management. 2008 (2) 78-79. (In Chinese)

[7] Leiqing Zhou, The situation and application of national sweeper, J. Traffic and transportation. 2008 (5) 88-89. (In Chinese)

[8] Shanmao Luo, Research and development of Chinese sweeper, J. Construction machinery technology and management. 2005 (5) 64-66. (In Chinese)

[9] Youlun Xiong, Robot technology, Huazhong University of Science and Technology press, Wuhan, 1996. (In Chinese)

[10] Chunlin Zhang, Advanced Kinematics and Dynamics of Mechanisms, Beijing Institute of Technology press , Beijing, 2006. (In Chinese)

[11] Xiangfeng Ma. Robot Mechanism, Machinery industry press, Beijing, 1991. (In Chinese)

[12] Tingli Yang, Theory and Application of Robot Mechanism Topology, Technology press,Beijing, 2012. (In Chinese)

[13] Yi Cao, Xiujuan Lee, Wei Ning, Computation and Geometrical Error Analysis of a 3D's work space, J. Mechanical science and technology. 2008 (2) 78-79. (In Chinese)

[14] Yanjiang Zhao, A method for solving robot workspace based on matalab, J. Mechanical science and technology for aerospace engineering, 2009,12 (28) 1658-1660. (In Chinese)

[15] Rastegar J, Fardanesh B, Manipulator Workspace Analysis Using the Monte Carlo Method, J. Mech. Mach.Theory, 1990, 25(2) 233-239. 